\title{
BatchServer: a web server for batch effect evaluation, visualization and correction
}

Tiansheng Zhu ${ }^{1,2,3,4}$, Rui Sun ${ }^{2,3,4}$, Fangfei Zhang ${ }^{2,3,4}$, Guo-Bo Chen ${ }^{5}$, Xiao Yi ${ }^{2,3,4}$, Guan Ruan $^{2,3,4}$, Chunhui Yuan ${ }^{2,3,4}$, Shuigeng Zhou ${ }^{1, *}$, Tiannan Guo ${ }^{2,3,4 *}$

${ }^{1}$ Shanghai Key Lab of Intelligent Information Processing, and School of Computer Science, Fudan University, China;

${ }^{2}$ Key Laboratory of Structural Biology of Zhejiang Province, School of Life Sciences, Westlake University, Hangzhou, Zhejiang, China;

${ }^{3}$ Westlake Laboratory of Life Sciences and Biomedicine, Hangzhou, Zhejiang, China;

${ }^{4}$ Institute of Basic Medical Sciences, Westlake Institute for Advanced Study, Hangzhou, Zhejiang, China;

${ }^{5}$ Clinical Research Institute, Zhejiang Provincial People's Hospital, People's Hospital of Hangzhou Medical College, Hangzhou, Zhejiang, China.

\section{Correspondence:}

Shuigeng Zhou: sgzhou@ fudan.edu.cn

Tiannan Guo: guotiannan@westlake.edu.cn 


\section{Table of Contents}

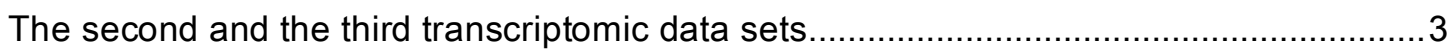

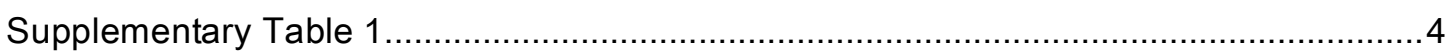

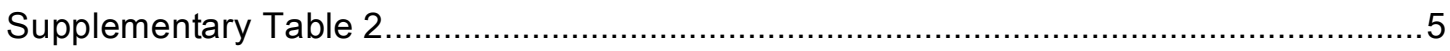

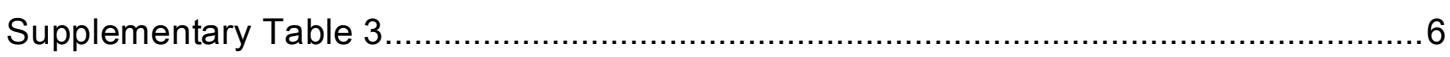

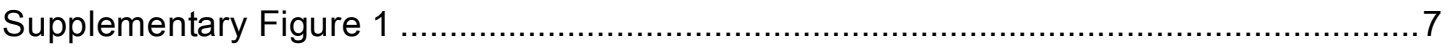

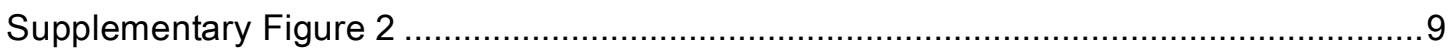

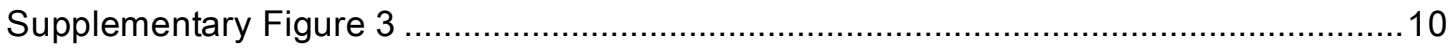

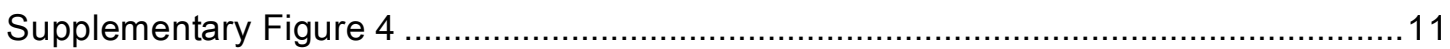




\section{The second and the third transcriptomic data sets}

The second transcriptomic data set came from Bioconductor package BatchQC sample data, which captured 89 samples when activating 9 different growth pathway genes (1,600 genes) in human mammary epithelial cells (GEO accession: GSE73628). The data contained three batches and ten different conditions. We did the same experiment on this data set as transcriptomic data set one. The performance of BatchServer were similar to first data set, and details in Supplementary Table 2 and Supplementary Figure 1.

The third transcriptomic data set was from Bioconductor library bladderbatch, a microarray gene expression data with 52 genes on 57 bladder samples in 5 batches. The time cost was presented in Supplementary Table 3. The results were also very similar to the first transcriptomic data set Supplementary Figure 2ABC. It was obviously to see from

Supplementary Figure 3 that when $\hat{\delta}$ was poorly fit non-parametric Bayes implementation of ComBat was in use.

The experiment of the three data sets indicate BatchServer were suitable for evaluating and removing batch effects and enhance biological signals. 


\section{Supplementary Table 1}

\begin{tabular}{llll}
\hline Parameter & User & System & Elapsed \\
\hline nonparametric & 3195.69 & 203.70 & 3400.65 \\
parametric & 0.53 & 0.11 & 0.64 \\
auto & 3209.11 & 239.84 & 3452.00 \\
\hline
\end{tabular}

Supplementary Table 1 Time consumed (seconds) for batch effect adjust using

autoComBat in transcriptomic data set 1 . The 'User' time is the CPU time charged for the execution of user instructions of the calling process. The 'System' time is the CPU time charged for execution by the system on behalf of the calling process, and the 'Elapsed' time is the 'real' elapsed time since the process was started (similarly hereinafter). 
Supplementary Table 2

\begin{tabular}{llll}
\hline Parameter & User & System & Elapsed \\
\hline nonparametric & 19.16 & 0.10 & 19.25 \\
parametric & 0.05 & 0.00 & 0.04 \\
auto & 18.20 & 0.25 & 18.50 \\
\hline
\end{tabular}

Supplementary Table 2 Time consumed (seconds) for batch effect adjust using autoComBat in transcriptomic data set 2. 
Supplementary Table 3

\begin{tabular}{llll}
\hline parameter & user & system & elapsed \\
\hline parametric & 0.26 & 0.00 & 0.26 \\
nonparametric & 6483.32 & 0.46 & 6488.14 \\
auto & 6577.83 & 0.76 & 6580.92 \\
\hline
\end{tabular}

Supplementary Table 3 Time consumed (seconds) for batch effect adjust using autoComBat in transcriptomic data set 3 . 


\section{Supplementary Figure 1}
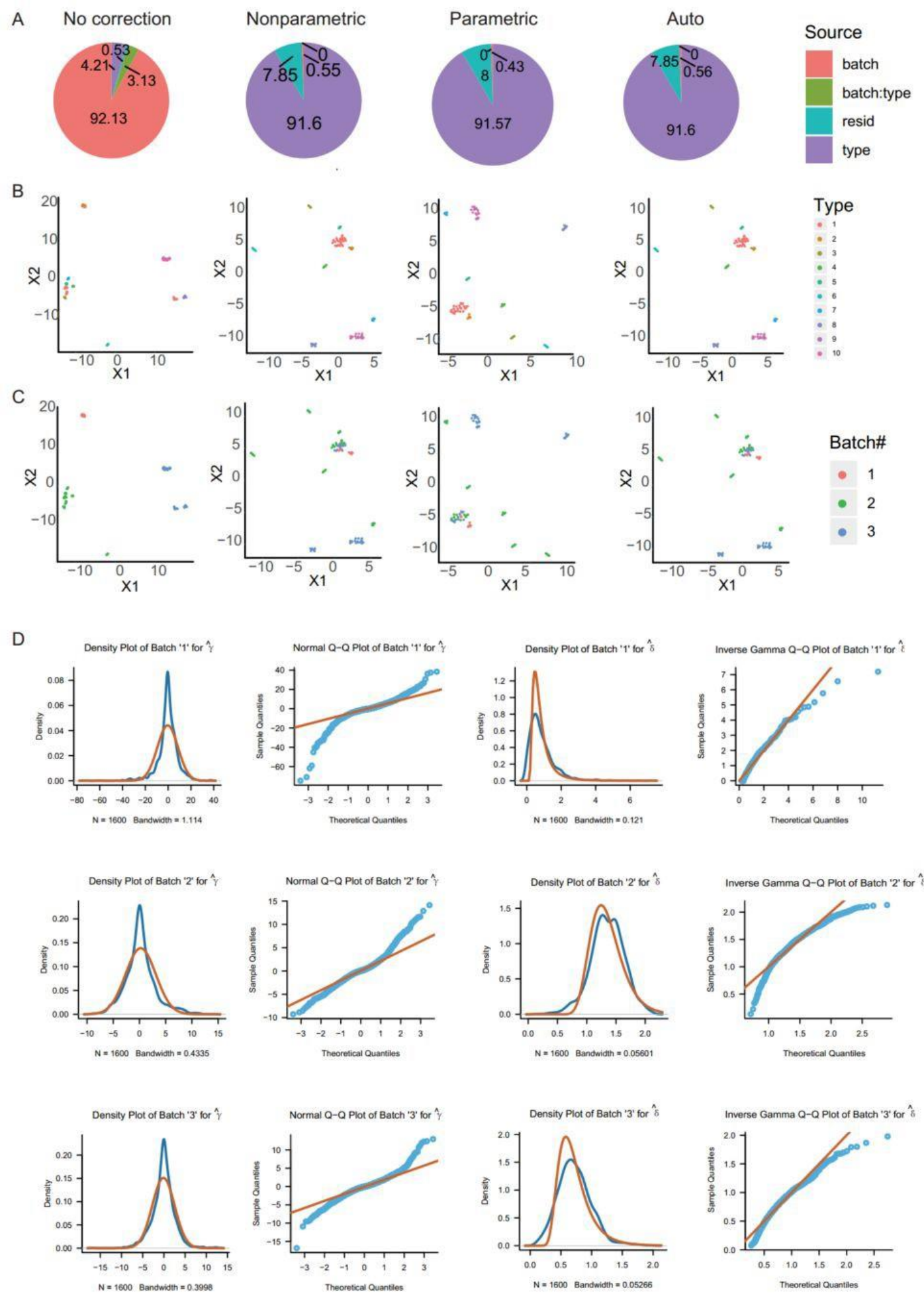

\section{Supplementary Figure 1 Performance of autoComBat by the second transcriptomic}

data set. A) Pie plots of batch effect using PVCA with no correction and par.prior set to nonparametric, parametric or auto for autoComBat. BC) UMAP plots show the biological and 
batch effect clustering, respectively. D) Prior plots of batch fitting effect by autoComBat. 


\section{Supplementary Figure 2}

A
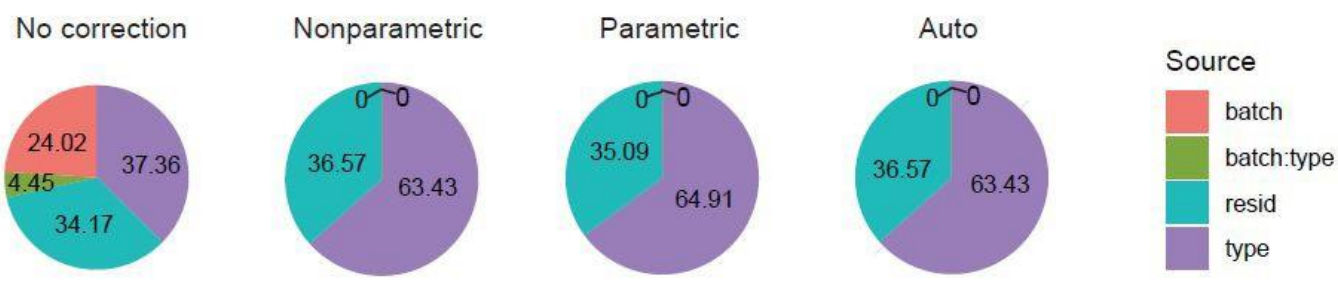

B
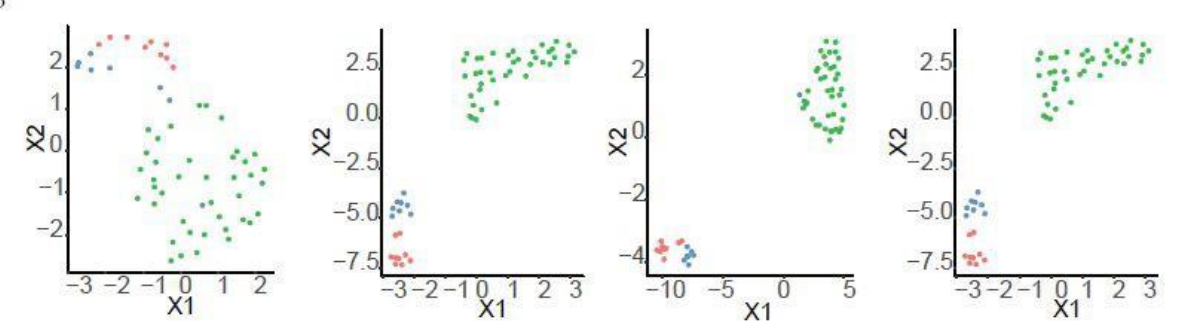

Type

- Biopsy

- Cancer

Normal

$\mathrm{C}$
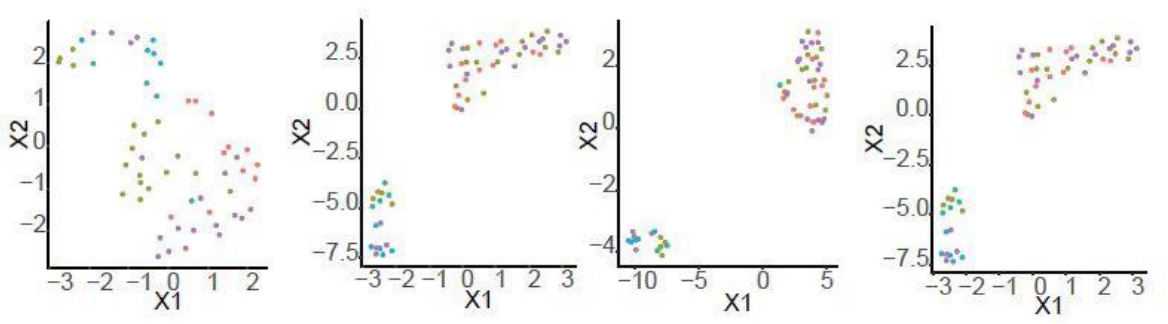

Batch\#

- 1

- 2

- 3

- 4

- 5

\section{Supplementary Figure 2 Performance of autoComBat by the third transcriptomic data}

set. A) Pie plots of batch effect using PVCA with no correction and par.prior set to nonparametric, parametric or auto for autoComBat. BC) UMAP plots show the biological and batch effect clustering, respectively. 


\section{Supplementary Figure 3}
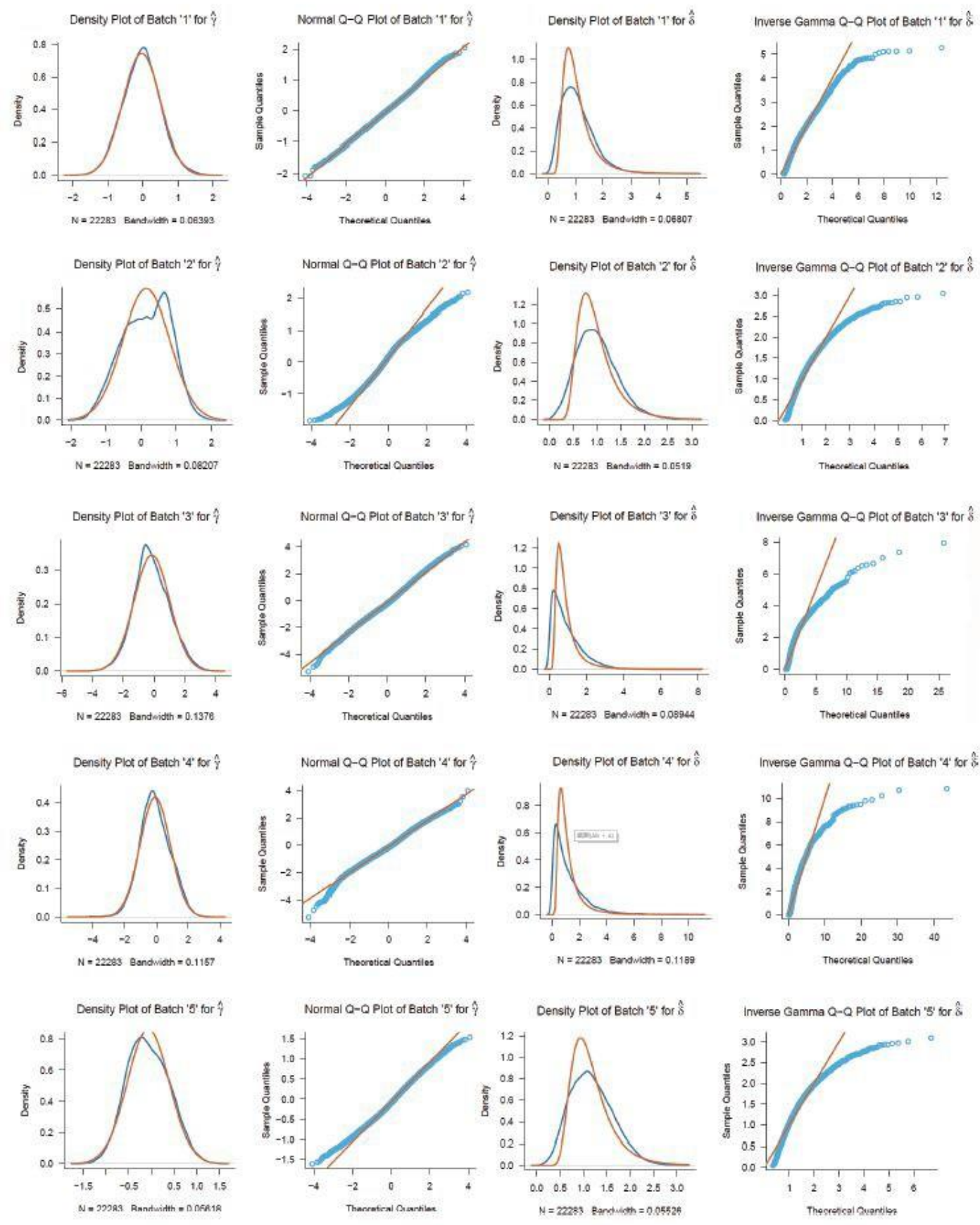

Supplementary Figure 3 Prior plot of batch fitting effect by autoComBat by the third transcriptomic data set. 
Supplementary Figure 4
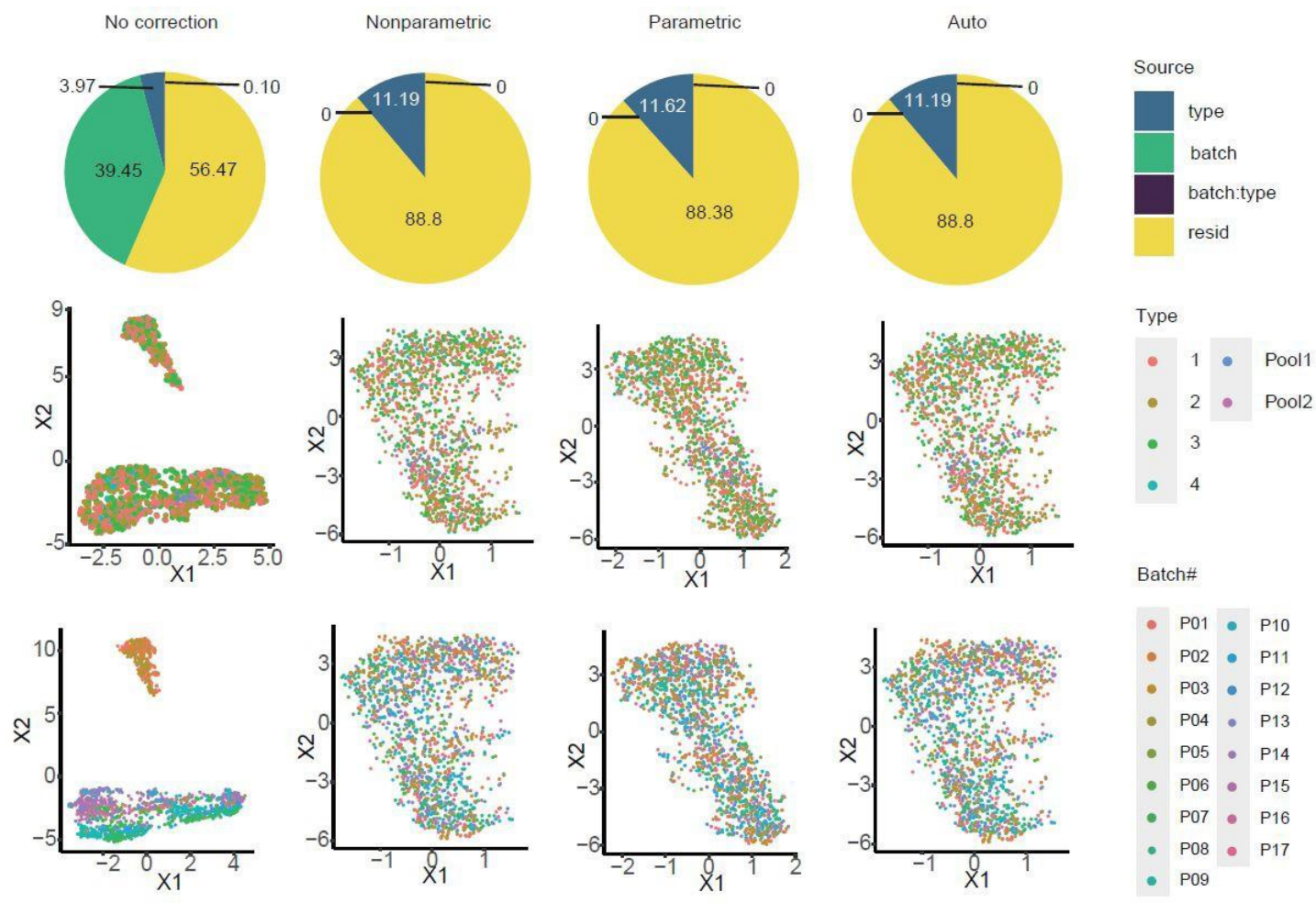

- P01 - P10

- P02 - P11

- P03 - P12

- P04 P13

- P05 P14

- P06 P15

- P07 - P16

- $\mathrm{P} 08$ - P17

Supplementary Figure 4 Performance of BatchServer by DIA-MS-based proteomics

data set. The first column shows PVCA and UMAP analysis of DiOGenes dataset. The second to fourth columns depict PVCA and UMAP after batch correction using autoComBat. 